\title{
Prospective Study of Autologous Adipose Derived Stromal Vascular Fraction Containing Stem Cells for the Treatment of Knee Osteoarthritis
}

\author{
Mark Berman, MD*, Elliot Lander, MD, Thomas Grogan, MD, Walter O'Brien, MD, \\ Jonathan Braslow, MD, Shawntae Dowell and Sean Berman, MS
}

Cell Surgical Network, University of Southern California, USA

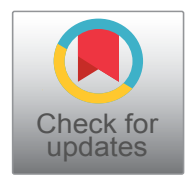

*Corresponding author: Mark Berman, MD, Conception and Design, Manuscript Writing: Final Approval of Manuscript, Provision of Study Material or Patients Cell Surgical Network, University of Southern California, 120 S. Spalding Dr. \#300, Beverly Hills, CA 90212, USA, Tel: 310-274-2789, Fax: 310-943-1960

\begin{abstract}
Background: The management of osteoarthritis of the knee runs the spectrum of care from a variety of conservative treatments often culminating in total joint arthroplasty. We initiated a large prospective study to evaluate whether autologous adipose derived stromal vascular fraction (SVF - rich in stem cells) therapy is a safe and effective option.
\end{abstract}

Methods: A patient funded prospective study of 2,586 patients from a network of physicians participated in an IRB approved study using autologous stromal vascular fraction SVF (ClinicalTrials.gov as NCT10953523). All patients were treated with a standardized surgical protocol to harvest lipoaspirate, isolate and deploy autologous SVF. Data was collected using an online registry and patients were followed-up via an automatic online database collection service.

Results: 2,586 patients were treated. Statistically significant improvement was seen at 1 and 2 years - meaning less pain and greater ease of mobility. There was no difference between male or female outcomes $(82 \%$ overall improvement). All BMI levels showed improvements though higher BMls had less improvement. There was no difference in outcomes between SVF alone or with PRP added to SVF. Improvement was the same regardless of payment or receiving free care. There were very few adverse events and those that did occur were largely very minor or easily treatable.

Conclusion: Deployment of autologous SVF represents a simple surgical procedure that can be safely performed in an adequate outpatient environment under straight local anesthesia and demonstrates very good outcomes even in difficult cases of chronic knee arthritis.

\section{Introduction}

Multiple peer-reviewed publications exist showing that adipose derived stromal vascular fraction containing adult mesenchymal stem cells may improve the condition of inflammatory knee conditions [1-5].

Animal studies show double-blind examples of significant improvements in using adipose derived stem cells for degeneration and injuries [6].

Multiple studies suggest that allogeneic stem cells may be efficacious and safe [7-11]. While an off-theshelf stem cell product might be idyllic, there are still long-term concerns about immunogenicity as allogeneic cells, while initially immune neutral, or immune evasive, will differentiate into the donor immune characteristics $[12,13]$. Autologous stem cells possess no risk of short or long-term allergic or immune response. Additionally, an allogeneic source of human stem cells must be free of any infectious agents. As humans are not grown in sterile environments, it's virtually impossible to assure society that allogeneic sources are devoid of possible prions or viruses that may go undetected. A variety of papers have already documented risks of disease transmission from a variety of allogeneic sources [14-18].

Patients presenting with painful knee osteoarthritis may benefit from receiving immediate point of care ad-

Citation: Berman M, Lander E, Grogan T, O'Brien W, Braslow J, et al. (2019) Prospective Study of Autologous Adipose Derived Stromal Vascular Fraction Containing Stem Cells for the Treatment of Knee Osteoarthritis. Int J Stem Cell Res Ther 6:064. doi.org/10.23937/2469-570X/1410064

Accepted: November 27, 2019: Published: November 29, 2019

Copyright: (c) 2019 Berman M, et al. This is an open-access article distributed under the terms of the Creative Commons Attribution License, which permits unrestricted use, distribution, and reproduction in any medium, provided the original author and source are credited. 
ipose stromal vascular fraction (SVF) surgically isolated from lipoaspirate. At the time of treatment, patients may also have the option of sending their autologous adipose tissues to a laboratory banking facility for cGMP production and storage of Mesenchymal Stem Cells (MSCs) for future use or repeat treatments. Yet, same day treatments with autologous cells (SVF) prevents the necessary wait time for cGMP laboratory preparation.

In order to evaluate the efficacy and safety of adipose-derived SVF for arthritic knee pain, an IRB approved outcomes study was initiated in 2012 through to 2015 using a standardized surgical procedure among a number of different Cell Surgical Network affiliated medical clinics that share protocols and techniques for SVF production and deployment. There was no placebo arm using sham surgery. Patients were queried via an automated email campaign to ascertain clinical outcomes following intravenous and intra-articular deployment of adipose-derived SVF. The initial goal was to learn if autologous SVF delivered using a simple point of care surgical procedure was safe and effective. Results were stratified and analyzed to determine optimal conditions.

\section{Materials and Methods}

An IRB approved (International Cell Surgical Society) outcomes study and IRB approved consent for osteoarthritis, including knees, was initiated in 2012 and registered with ClinicalTrials.gov as NCT10953523. Patients over 18 years of age that had complaints of knee arthritis were considered for therapy. Patients were not limited to any particular Kellgren-Lawrence scale. Patients with active systemic infections or dental infections were excluded. Most patients had already tried and failed to improve with a variety of interventions including NSAIDS, steroid injections, platelet rich plasma injections, rest, heat, cold, magnetic devices, hyalurons and even arthroscopic procedures- none of which provided sustained relief beyond a few weeks resulting in reduced mobility. Data was collected from all participating affiliates of the Cell Surgical Network (CSN) through an HIPAA compliant online database (TrackVia.com). CSN affiliate sub-investigators that contributed to this study are listed in the acknowledgement section.

CSN affiliates who were not orthopedic specialists themselves were encouraged to work in multi-disciplinary teams in order to have an orthopedic or sports medicine specialist involved in the care and follow-up of the patients. Many affiliates from other specialty background, such as pain management or family practice, etc., by virtue of their education, training and experience, felt qualified evaluating, treating and following the patients themselves.

All patients underwent a uniform mini-liposuction procedure consistent with the CSN IRB approved protocol. This included a specific method for providing a sub-dermal local anesthesia to maximize comfort, safety, and limit exposure of stem cells to potentially cytotoxic levels of lidocaine. Syringe lipo-aspiration was accomplished using specially developed syringes (Medikan, South Korea). $50 \mathrm{ml}$ of lipoaspirate was recovered from one of several potential patient harvest sites. The site depended on a variety of issues - doctor preference, patient preference, cosmetic result, consideration for possibly more optimal stem cell sites and ultimately the abundance of harvestable adipose tissue.

The lipoaspirate was condensed by centrifugation $(2,800 \mathrm{rpm}$ for 3 minutes) and the infranatant cells (approximately 1-2 $\mathrm{ml}$ because a number of cells are already mechanically dissociated) and approximately up to $25 \mathrm{ml}$ of condensed fat were transferred to another specialized syringe (TP102 Medikan, South Korea) along with $25 \mathrm{ml}$ of 12.5 Wunsch units of GMP collagenase (Roche) in normal saline for incubation with gentle agitation at $37{ }^{\circ} \mathrm{C}$ for $30-40$ minutes. Following this, the lipoaspirate was centrifuged for 4 minutes at $200 \mathrm{rcf}$ (relative centrifugal force - approximately 1,100 rpm). With unique equipment, the supranatant fluids were removed while maintaining the infranatant solution within the syringe. This prevented air exposure to the SVF preparation and allowed introduction of D5LR in order to dilute the collagenase by washing to imperceptible levels. Three dilutions were completed leaving 4-10 ml of concentrated cells at the bottom of the syringe. This solution was transferred to a $10 \mathrm{ml}$ luer lock syringe and filtered through a 100-micron nylon filter producing 4-10 $\mathrm{ml}$ of final SVF isolate.

The affected knee or knees were injected with 3-5 $\mathrm{ml}$ of SVF solution. This was most frequently done by injection either medially or laterally just above the tibial plateau and deep into the joint. A wheal of local anesthesia or ethyl chloride spray was provided for skin anesthesia. A 22 g, 1.5 inch needle was most often used for the deployment into the joint. Most physicians performed the procedure in the office or surgical treatment room without aid, however, in some cases, ultrasound or fluoroscopy were employed. Most often the remaining solution was provided via an intravenous infusion. In some cases, doctors chose to add Platelet Rich Plasma (PRP) to the intra-articular deployment. There have been a plethora of studies suggesting PRP brings a variety of important growth factors to the injury and may complement the addition of SVF. Physicians and patients were allowed to make the decision whether or not they wanted to add PRP and results were appropriately stratified.

Due to the high number of patients included in this particular study, responses to the WOMAC questionnaire proved futile. Questionnaire response rates improved significantly when patients recorded a simple visual acuity pain score of 0 (no pain) to 10 (worse pain they could tolerate) for 1) At rest, 2) Standing, 3) 
Table 1: When queried whether the patient experienced improvement (measured as a decrease in pain or improvement in perceived function - mobility - versus no improvement in pain or mobility) the above graph revealed the following:

Baseline total - 2,586 patients

1. 1 month - 1,643; Improve - 1,150 (69.99\%); Not improve - 408

2. 3 month - 1,330; Improve - 1,027 (77\%); Not improve - 226

3. 6 month - 1,097; Improve - 862 (78.58\%); Not improve - 154

4. 9 month - 803; Improve - 642 (79.95\%); Not improve - 96

5. 1 year - 615; Improve - 495; (80.49\%); Not improve - 69

6. 1 year 3 months - 363; Improve 293 (80.72\%); Not improve - 34

7. 1 year 6 months - 248; Improve - 198 (79.84\%); Not improve - 25

8. 2 years - 212; Improve - 165 (77.83\%); Not improve - 23

9. 3 years -57 ; Improve $-47(82.46 \%)$; Not improve -7

10. 4 years - 15; Improve - $13(86.67 \%)$; Not improve - 1

11. 5 years - 4 ; Improve - $4(100 \%)$; Not improve -0

Walking and 4) Running. An automatic program through Trackvia (Denver, CO) to contact and query our patients for follow-up responses at intervals of 1 week, 3 months, and then every 3 months until 36 months ( 3 years) and the yearly for the next two ( 4 and 5 ) years was used. Patients were asked to note their visual acuity pain scores each time and provide an overall opinion as to whether they had sustained improvement in function (i.e. mobility) without or with decreased or tolerable pain compared to before the received deployment. Pain scores are reasonably objective while improvement based upon patient response remains subjective.

Prior to SVF deployment, sub-investigators measured total cell counts using the Countess device (Invitrogen) that quantified cells over 10 microns in size (to exclude RBCs). Results ranged from 10 to 300 hundred million cells. Viability was also checked using $0.4 \%$ trypan blue and ranged from $65-95 \%$. Cell counts varied significantly among patients as expected with an autologous biologic. SVF cannot currently be isolated at point of care uniform in dose, strength and purity due to the wide variety and proportion of cells in the final isolate. Stem cell counts were not obtained on study patients however as pre-clinical work, 50 SVF samples were sent for flow cytometry to a collaborative research lab (i.e. Stem Immune) at UC San Diego and large but variable quantities of several types of stem cells were identified in each sample.

Flow cytometry analysis showed that freshly isolated SVF was heterogeneous and harbored four major subsets specific to adipose tissue:

1. CD34 high, CD45-, CD31-, CD146- adipose-derived stromal/stem cells (ADSCs),

2. CD34 low, CD45+, CD206+, CD31-, CD146- hematopoietic (or angiogenic) stem cell-progenitors (HSC-progenitors),

3. CD34 high, CD45-, CD31+, CD146+ adipose tissue-endothelial cells, and

4. CD45-, CD34-, CD31-, CD146+ pericytes [19].
Statistics were determined using the ANOVA method

\section{Results}

2,586 patients participated in SVF deployment. There was a drop-off in data collection even at one month to 1,643 patients. Overall, patients reported that they had improvement with pain or function in nearly $80 \%$ of the cases. There were a few patients reporting 4 and 5 years later that they still maintained good results, but these numbers were obviously few (See Table 1). There were statistically significant improvements at 1 and 2 years respectively based upon $\mathrm{n}=615$ and $\mathrm{n}=212$ patients reporting. Improvement was determined subjectively by patients both based upon actual decrease in pain and their impression of whether they improved with respect to pain and function (i.e. mobility). A typical standing knee $x$-ray often revealed a lack of joint space and with improvement, an increase in joint space was often seen suggesting possible cartilage re-growth (Figure 1). The study stratified the patients and reported results based upon averages among all patients. Many patients were restored to pain free activities. Many younger patients were able to resume complete competitive sporting activities, while many older patients were content to be able to walk without pain so they could resume travel, golf and normal daily routines.

Patients were stratified into groups according to their pain level as 7-10 or 1-6. 1,017 patients were entered in the 7-10 pain level for walking. Figures represent patients followed for walking pain up to two years. Mean baseline pain level for the left and right knee respectively, was 7.308 and 7.388. At one year, 154 left knee patients reported pain levels at an average of 3.052 and 151 right knee patients reported pain levels averaging 3.305. This was considered statistically significant (Figure 2).

1,404 patients were entered into the 1-6 pain category for walking. Their mean baseline pain level for the left and right knee respectively, was 4.285 and 4.324 . At one year, 213 left knee patients reported an average of 


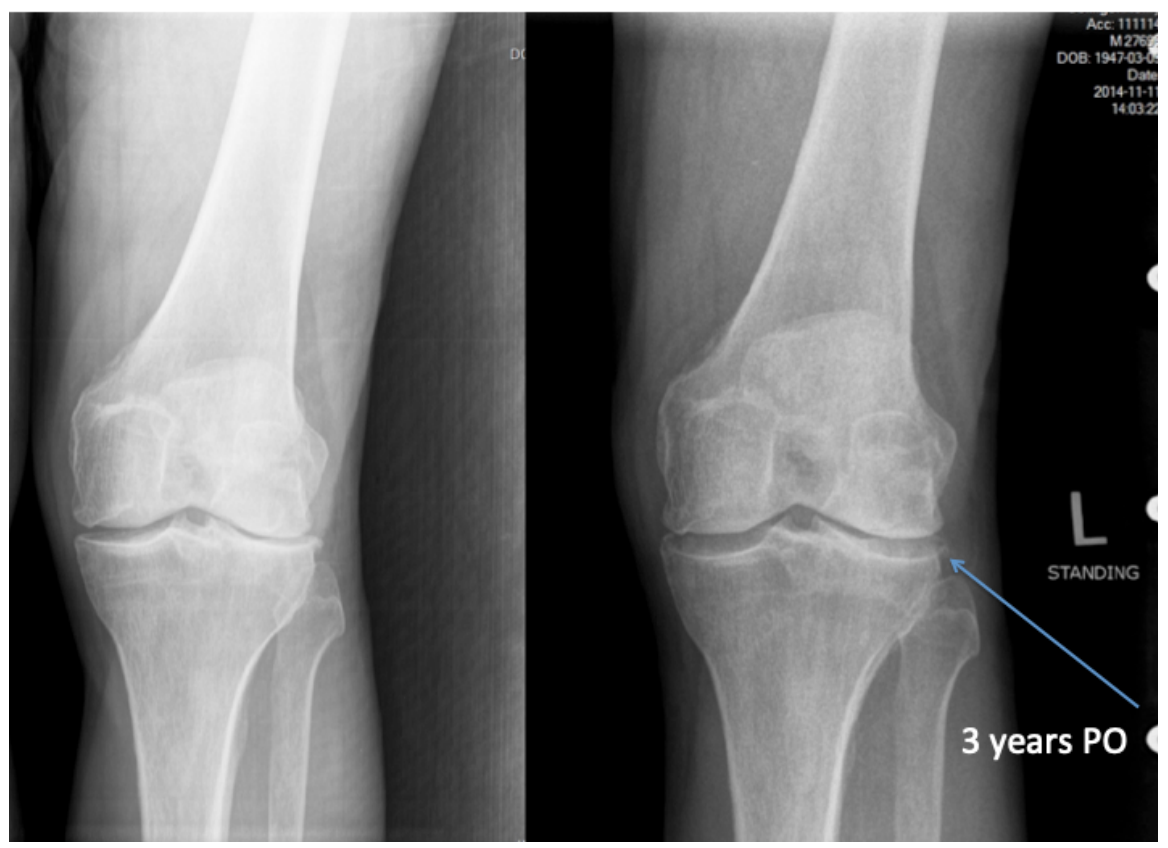

Figure 1: Typical knee patient pre-deployment and at 3 years post-deployment of SVF only.

\section{Walking Baseline Pain 7-10}

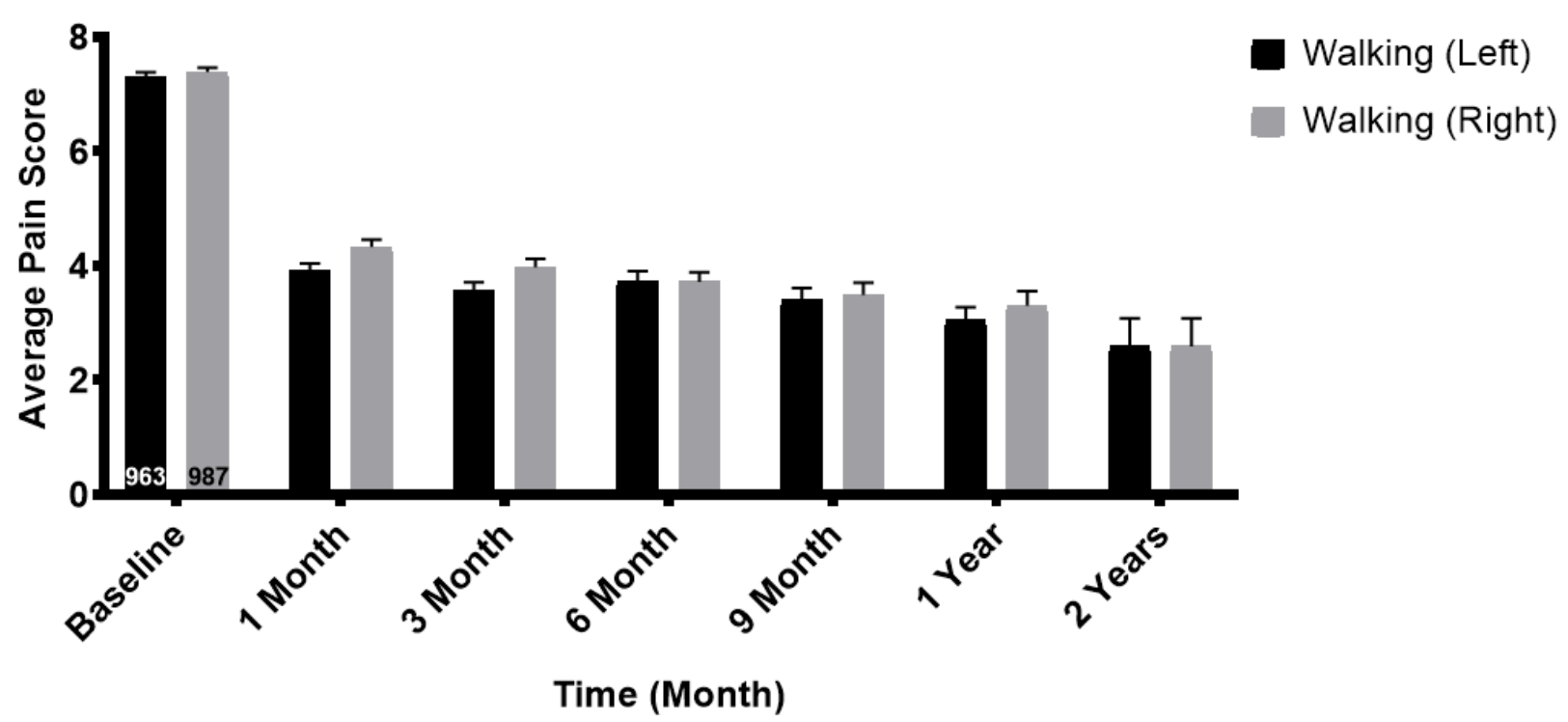

Baseline Left Mean \pm SEM: $7.308 \pm 0.07248$

1 Year Left Mean \pm SEM: $3.052 \pm 0.2243$

$\mathrm{n}=154 \mathrm{P}$ Value: $<0.0001$, Significantly different $(\mathrm{P}<0.05)=$ yes

2 Year Left Mean \pm SEM: $2.5950 \pm 0.4893$

$n=37 P$ Value $:<0.0001$, Significantly different $(P<0.05)=y e s$

Baseline Right Mean \pm SEM: $7.388 \pm 0.07174$

1 Year Right Mean \pm SEM: $3.305 \pm 0.2516$

$n=151 P$ Value: $<0.0001$, Significantly different $(P<0.05)=y e s$

2 Year Right Mean \pm SEM: $2.5950 \pm 0.4868$

$n=42 P$ Value: $<0.0001$, Significantly different $(P<0.05)=y e s$

Figure 2: 1,950 knees with statistical significance at 1 and 2 years.

2.113 and 215 right knee patients reported pain levels averaging 2.260. This was considered statistically significant (Figure 3). All age groups (from below 30 to over 80) posted results consistent with the overall trends.
There did not appear to be any age category that did better or worse than another. In general, most patients started with walking pain in the 4-5 range on average and after 1 year the average of 1-2 pain score for walk- 


\section{Walking Baseline Pain 1-6}

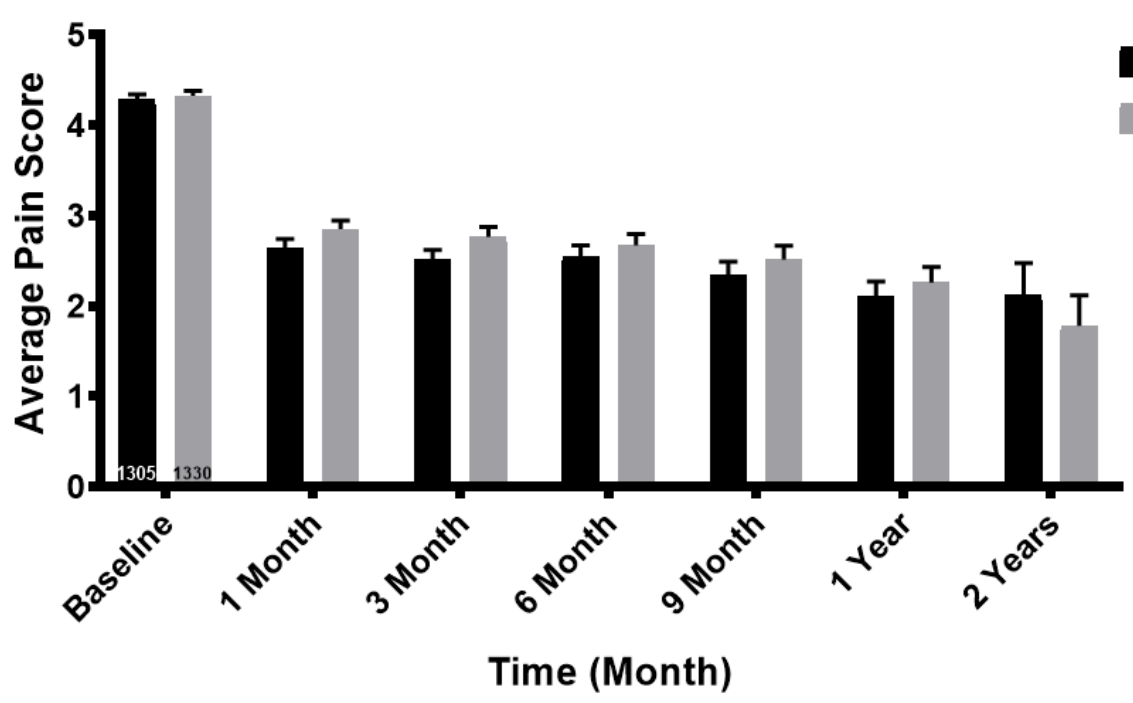

Walking (Left)

Walking (Right)

Baseline Left Mean \pm SEM: $4.285 \pm 0.0561$

1 Year Left Mean \pm SEM: $2.113 \pm 0.1581$

$n=213, P$ Value: $<0.0001$, Significantly different $(P<0.05)=y e s$

2 Year Left Mean \pm SEM: $2.1220 \pm 0.3543$

$n=49, P$ Value: $<0.0001$, Significantly different $(P<0.05)=y e s$

Baseline Right Mean \pm SEM: $4.324 \pm 0.05713$

1 Year Right Mean \pm SEM: $2.260 \pm 0.1729$

$n=215, P$ Value: $<0.0001$, Significantly different $(P<0.05)=y e s$

2 Year Right Mean \pm SEM: $1.78 \pm 0.3372$

$n=50, P$ Value $:<0.0001$, Significantly different $(P<0.05)=y e s$

Figure 3: 2,635 knees with statistical significance at 1 and 2 years.

BMI 18.5-25

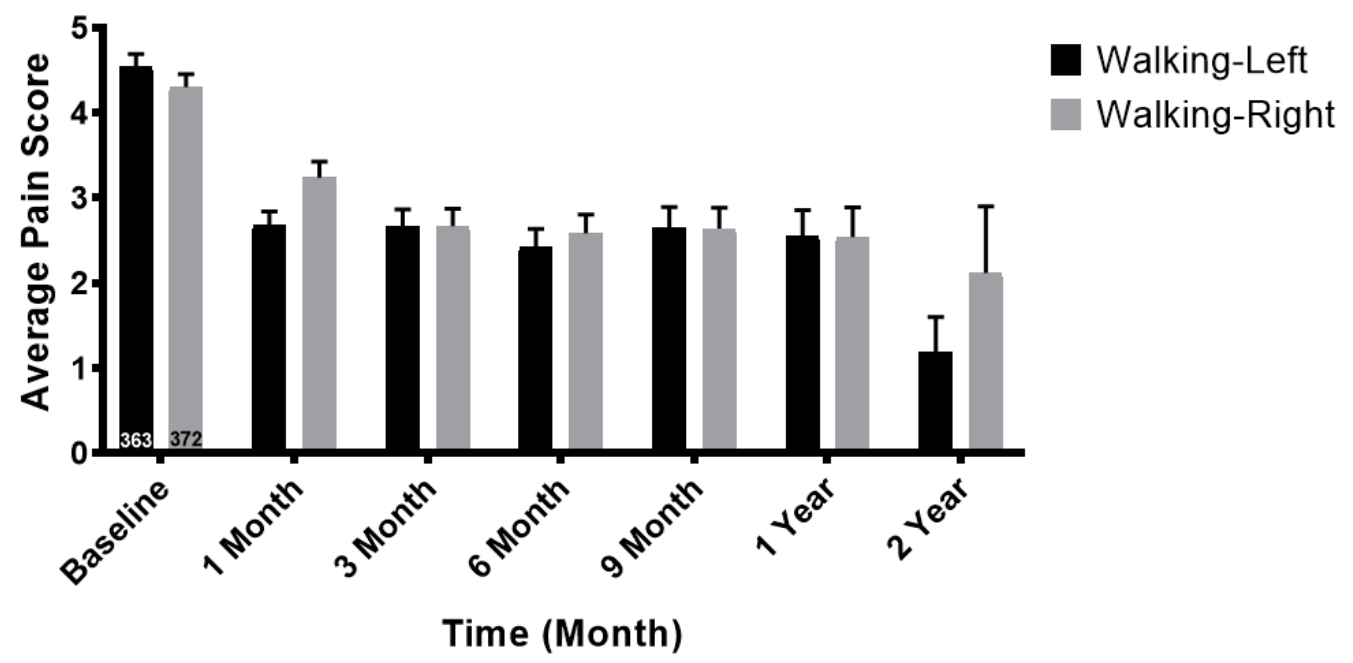

Baseline Left Mean \pm SEM: $4.537 \pm 0.1501$

1 Year Left Mean \pm SEM: $2.545 \pm 0.307$

$n=66, P$ Value $:<0.0001$, Significantly different $(P<0.05)=y e s$

2 Year Left Mean \pm SEM: $1.188 \pm 0.4105$

$n=16, P$ Value $:<0.0001$, Significantly different $(P<0.05)=y e s$

Baseline Right Mean \pm SEM: $4.298 \pm 0.1525$

1 Year Right Mean \pm SEM: $2.537 \pm 0.3477$

$n=67, P$ Value: $<0.0001$, Significantly different $(P<0.05)=y e s$

2 Year Right Mean \pm SEM: $2.118 \pm 0.7807$

$n=17, P$ Value: 0.0031 , Significantly different $(P<0.05)=y e s$

Figure 4a: Statistically significant improvement in normal BMI. 
BMI 30+

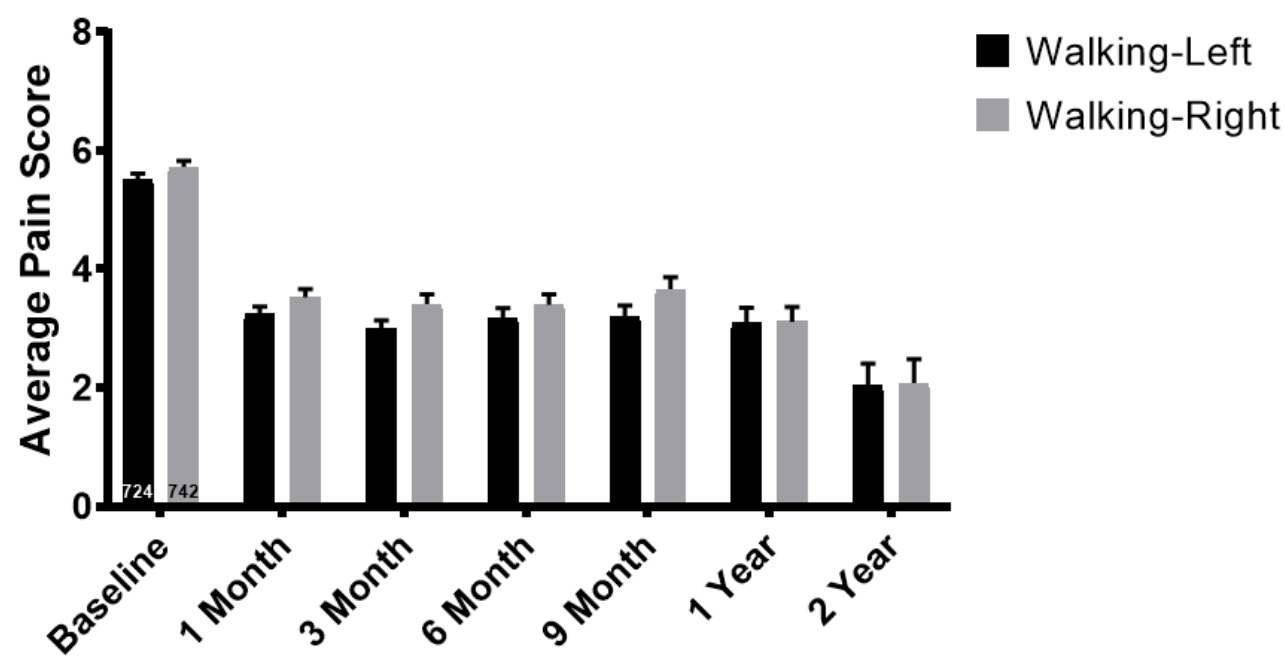

Time (Month)

Baseline Left Mean \pm SEM: $5.504 \pm 0.1055$

1 Year Left Mean \pm SEM: $3.091 \pm 0.2607$

$n=132$, $P$ Value: $<0.0001$, Significantly different $(P<0.05)=y e s$

2 Year Left Mean \pm SEM: $2.032 \pm 0.3753$

$n=31, P$ Value $:<0.0001$, Significantly different $(P<0.05)=y e s$

Baseline Right Mean \pm SEM: $5.722 \pm 0.1035$

1 Year Right Mean \pm SEM: $3.114 \pm 0.2461$

$n=149, P$ Value: $<0.0001$, Significantly different $(P<0.05)=y e s$

2 Year Right Mean \pm SEM: $2.079 \pm 0.4034$

$n=38, P$ Value $:<0.0001$, Significantly different $(P<0.05)=y e s$

Figure 4b: Statistically significant improvement even with 30+ BMI though less compared to normal weight.

SVF vs SVF/PRP

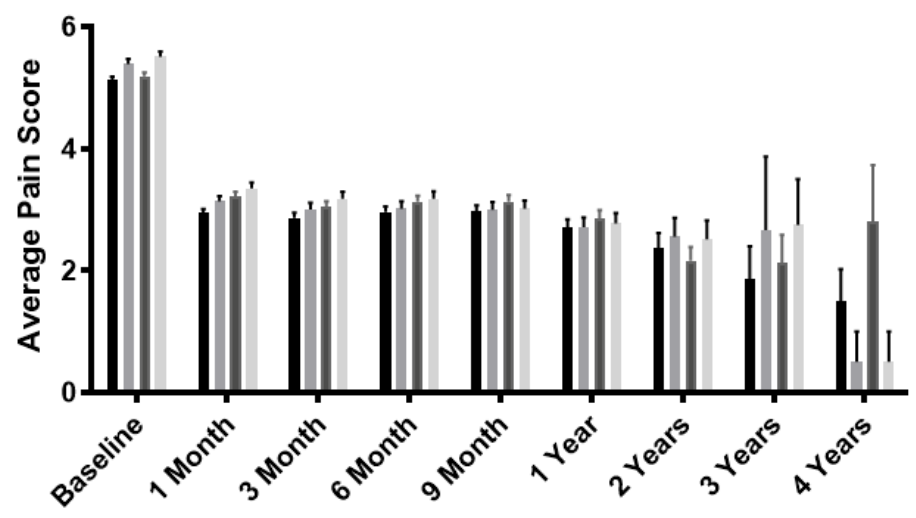

Time (Month)
SVF Only-Walking (Left)

SVF \& PRP-Walking (Left)

SVF Only-Walking (Right)

SVF \& PRP-Walking (Right)

\section{SVF Only}

Baseline Left Mean \pm SEM: $5.122 \pm 0.06054$

1 Year Left Mean \pm SEM: $2.705 \pm 0.1372$ $\mathrm{n}=403$

$P$ Value: $<0.0001$, Significantly different $(P<0.05)=$ yes

Baseline Right Mean \pm SEM: $5.19 \pm 0.06066$

1 Year Right Mean \pm SEM: $2.846 \pm 0.1451$

$n=415$

$P$ Value: $<0.0001$, Significantly different $(P<0.05)=$ yes
SVF \& PRP

Baseline Left Mean \pm SEM: $5.392 \pm 0.08306$

1 Year Left Mean \pm SEM: $2.715 \pm 0.1575$ $\mathrm{n}=284$

$P$ Value: $<0.0001$, Significantly different $(P<0.05)=y e s$

Baseline Right Mean \pm SEM: $5.51 \pm 0.08277$

1 Year Right Mean \pm SEM: $2.774 \pm 0.1681$

$\mathrm{n}=288$

$P$ Value: $<0.0001$, Significantly different $(P<0.05)=y e s$

Figure 5: No significant difference when comparing SVF alone versus SVF with PRP. 
ing was seen across all age categories.

There was no significant difference between the overall results between men ( $n=1,907$ knees) and women ( $n=1,848$ knees). Both reported improved results just above $82 \%$ on average for all times reporting, with statistically significant results at 1 and 2 years.

When patients were stratified by body mass index (BMI) there was improvement for most patients in all categories. Patients with higher BMI, particularly over 30, had less improvement compared to lower BMI patients, yet still reported improvements on average. While the trends were the same in all categories, we demonstrate BMI 18-25 (i.e. normal range) compared to $\mathrm{BMI} 30+$ (very overweight or obese) (Figure $4 \mathrm{a}$ and Figure $4 b)$.

Some investigating physicians used PRP as a supplement to SVF injected into the knee joint. Comparison of SVF alone to SVF supplemented with PRP revealed no significant difference in the overall results at the end of one year. Nearly all patients in both of these categories received supplemental IV infusion (Figure 5). SVF patients $(n=818$ experienced a baseline walking average pain of 5.17 and a 1-year walking pain decreased to an average of 2.75. SVF and PRP combined patients ( $\mathrm{n}$ = 572) experienced a baseline walking average pain of 5.35 and a 1-year walking pain decreased to an average of 2.74 .

Adverse events were mainly due to pain around the liposuction site and very occasionally related to the knee with the main complaint being increased swelling shortly after the procedure (Figure 6).

There were no serious adverse events such as death, hospitalization, infections, pulmonary emboli or other serious thrombus formation. A few patients thought they had increased pain and about $18 \%$ of the overall patients did not report improvement. Lack of improvement may be a risk but not an adverse event. A number of these patients went on to have total knee replacement. We do not have specific statistics on their outcomes following total arthroplasty. The most common problem was near immediate knee swelling and pain (0.28\%). When this occurred there was never a positive culture and whenever steroids were provided (dexamethasone 4-10 mg given as soon as possible into the tender edematous joint), there was a uniformly positive response. All cases had favorable responses and the patients went on to have good improvement without needing further treatments. One patient erroneously reported that they sustained a serious knee infection and eventually needed a total arthroplasty. The non-CSN affiliated physician that saw her the following day unfortunately drained the patient's knee without providing steroids, sent the specimen for culture that proved to be negative and injected the joint with bupivacaine.
In assessing possible tumor or cancer formation, few patients responded positively that they had cancers or benign tumors over a course of time. Most cancers or tumors occurred more than 18 months following the procedure. Some lesions, particularly skin cancers, had previously occurred and were reported months later occurring again. None of the tumors or cancers were considered to have been caused by the SVF and nor did they occur in any frequency greater than would be expected in the general population.

All other adverse events were not considered noteworthy.

Cell counts were done on all specimens collected however they weren't correlated with flow cytometry so there was no way of gauging any value in the data. When cells were counted using either size (e.g. cells between 10 and 60 micron) or structural elements (e.g. nucleated cells) to exclude red blood cells, counts varied from 30 to 800 million cells overall. Most cell counts ran around 50 - 100 million cells overall. Evaluation by flow cytometry of 50 samples of cells suggested that stem cell populations were approximately $10 \%$ of our overall cell count, but this didn't appear to be statistically significant. Overall cell viabilities ran between 70 and $90 \%$ remaining fairly constant throughout the data collection.

\section{Discussion}

By working together as a clinical research network, using the same equipment, same methods of anesthesia and surgical preparation, data was gathered from over 80 independent clinical sites affiliated with the Cell Surgical Network. Most clinics worked with a multispecialty team that included orthopedic surgeons. Patients self reported subjective outcomes data into the online $\mathrm{HI}$ PAA compliant database directly allowing the network to collect safety and efficacy data from all clinics. A significant amount of information collected through the database and analyzed demonstrated relative uniformity throughout the network. These CSN physician teams deserve considerably more gratitude and acknowledgment than the simple table we have included below in the Acknowledgement section.

As previously noted in safety studies, SVF is shown to be exceedingly safe [20]. This knee study exceeds the number of patients analyzed in previous safety studies and certainly corroborates the safety of autologous SVF for deployment to the knees along with an IV infusion. Nearly all of the 2,586 patients received supplemental intravenous infusions and IV infusion is currently considered part of the best practices protocol.

There were no serious adverse events (e.g. death, hospitalization, emboli, infections from SVF, etc.) directly related to receiving SVF. There were some adverse events reported by the patients with the most notable being related to knee swelling within 24 hours of the procedure. In some cases it was quite painful, but in all 


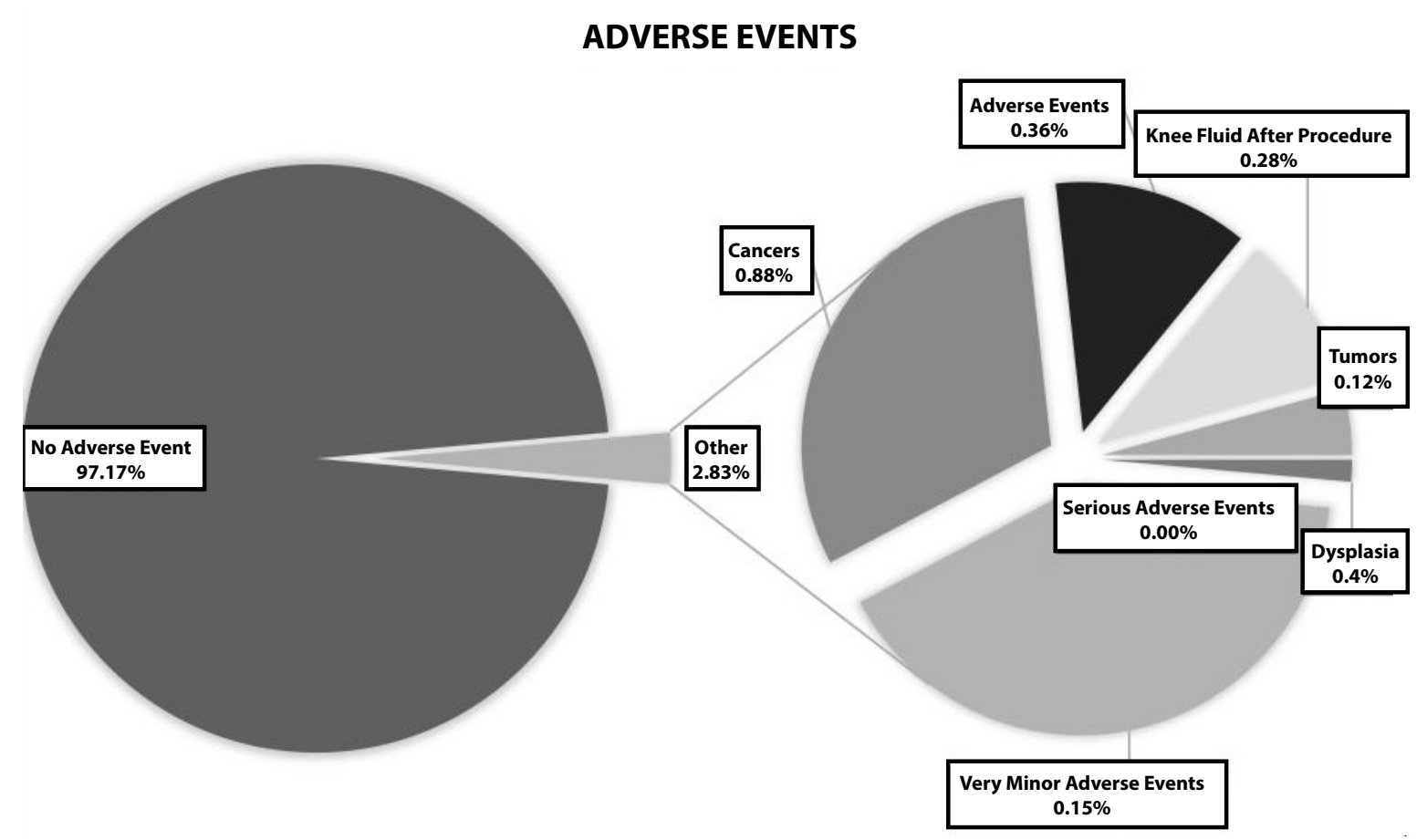

Figure 6: Adverse events were categorized as follows:

1. Serious Adverse Events (e.g. death, hospitalization, emboli, infections) $=0$.

2. Adverse Events $=9$ patients. $35 \%$ of total

a) Increased pain - 7

b) Rash (temporary) over whole body - 1

c) Periodically unstable -1 .

3. Knee fluid after the procedure $=7$ patients $0.28 \%$ of total

a) 3 drained

1. Cultures - negative -3

2. One received bupivicaine, later steroids and later had total knee replacement - erroneously self-reported an infection but cultures were all negative

3. One had steroids after drainage

b) 4 not drained, received steroid injection and improved.

4. Tumors or cancers reported: Total $C A=22$; Tumors $=3$; Dysplasia $=1-1 \%$ of total
a) Tonsil CA - 1
b) Uterine CA - 1 (6 month post)
c) Cervical dysplasia (18 months later)
d) Basal Cell CA - 8 (9 months - 2 years later)
e) Squamous Cell CA - 1
f) Breast CA - 4 (4 months later $\times 1$; others $2 \frac{1}{2}-3$ years later)
g) Prostate CA - 4 ( average 15 months later)
h) Renal CA - 1 (15 months later)
i) Sarcoma - 1 (15 months later)
j) Pancreatic CA - 1
k) Parathyroid tumor - 1 (18 months later)
l) Lipoma - 2 (9 month and 3 years later).

5. Very Minor Adverse Events (all temporary events) $1.1 \%$ of total
a) Liposuction defect complaints - 6
b) Liposuction site pain, itching, minor swelling, discomfort (temporary) - 14
c) No improvement, waste of money, felt they were mislead - 2
d) Increased weakness, tiredness - 2 at 1 month
e) Body aches, fever, night sweats - 3 (1 each) at 1 - 3 months later

Leg cramp, spasms - 2 (1 each). 
cases, when treated with an injection of dexamethasone $4 \mathrm{mg}$ intra-articular, it quickly resolved (within minutes or hours). None of them were infected (no positive cultures). Most adverse events were related to pain around the liposuction site and that was generally minimal (responded to temporary pain medication) and transient in all cases. The reported incidence of cancer or benign tumors generally occurred much later than one would expect in a causal relationship or previously existed (e.g. skin cancers) prior to treatments. The incidence of cancers was not any greater than would be expected in this general population and would therefore not be attributable to autologous SVF.

These prospective surgical procedures were done as part of an Institutional Review Board (organized through the International Cell Surgical Society) approved study. As a surgical procedure it was important to gather data to look for safety as well as efficacy. As a surgical procedure with an abundance of publications suggesting efficacy, a patient registry was established for prospective data rather than trying to set up a placebo trial. The amount of statistically significant data suggests that SVF is efficacious for knee arthritis amongst a variety of patients. Fairly uniform results were observed across all age groups. Patients with BMI above 30 faired less well than those below 30 however, all groups showed overall positive responses and decreasing pain scores. There was no significant difference between men and women.

When comparing clinical outcomes where PRP was added to the SVF versus SVF deployment alone, no statistical differences were observed in related pain outcomes. SVF is a combination of many cells including platelets, growth factors, and extracellular matrix materials. At least four varieties of adult stem cells have been identified in SVF with the two largest groups noted by CD34 and CD45 markers. Respectively, these represent preadipocytes and angiogenic cells found within the lining of the larger blood vessels [19].

Patients exhibited a positive response to SVF clinically for what appeared to be fairly long-term compared to other currently available injectable remedies. Most of the patients in this study presented for SVF deployment prior to requiring a total knee replacement procedure. The majority had exhausted all conventional treatments for knee arthritis such as NSAIDs, steroid injections, platelet rich plasma, hyaluronic acid injections, and even arthroscopic intervention. Since there was no lasting improvement from any of these previous treatments, it is suggested that none of the patients had any lasting affects from a possible placebo effect of those treatments. Since most (over $80 \%$ ) of the SVF treated patients sustained significant lasting improvement (greater than 1 year), it could be suggested that SVF may have a (much) better placebo effect than any of the other previous treatments.
Several of the patients continue to experience positive results as long as 8 years after their initial SVF injection (many prior to the official start of the study group). Since the patients are made up of cells and will continue to be exposed to normal cellular degeneration, the suggestion remains that repeated cell therapy should likely be necessary as time and natural degeneration or arthritic disease progression goes on.

Patients were either called for follow-up or in most cases contacted automatically via a software follow-up program securely integrated with the database. Patient responses were made in the comfort of their own environment without a physician looking over their shoulder or possibly being able to influence their response (i.e. Hawthorne effect). Nonetheless, patient compliance in responding decreased over time. 2,586 patients were enrolled in the study, but only 1,643 responded at the first month and 615 patients at the end of one year. Still, the percentage of patients showing improvement remained relatively stable with a statistical significance.

In a few cases where patients did not respond positively, arthroscopy was performed to evaluate their condition. In each case, at least one of the articular surfaces was devoid of any cartilage. For this reason, it is suspected that if there is no cartilage remaining on an articular surface, then there will be no adequate cellular signals to prompt the SVF (i.e. stem cells) to initiate repair of the arthritic condition.

It is important to note that the patients in the study were routinely charged for their surgical procedure. In many cases, patients were treated at no cost or markedly reduced cost depending upon any number of personal circumstances. This study had no investment or grant funding and as such patient funding was relied upon in order to subsidize those with less economic means. No difference was seen in response between patients that paid for their procedure versus those that paid nothing or had reduced fees.

Analysis of the study's patient population showed that positive results from SVF deployment were largely dependent upon the state of the injury. Most of the patients in the study opted to receive SVF in hopes of avoiding total joint replacement and thus, they exhibited chronic conditions that left them with very little or no cartilage(apparent by $\mathrm{x}$-ray) in their joint. Increased resolution of pain and cartilage repair should be achieved by providing cell therapy for patients during an earlier acute phase rather than the more chronic advanced phases of their condition, common in this study. Early on, there is likely to be more cartilage present, a strong and pronounced cytokine response, and potentially a requirement for fewer stem cells and reduced time for healing. 
The SVF isolation procedure is a simple surgical procedure whereupon physicians isolate autologous cells from adipose tissue both through mechanical disruption (e.g. liposuction alone causes disruption of cells from adipose [21]) and other advanced means (e.g. enzymatic digestion of collagen to further free cells from adipose tissue), then redeploy these isolated cells into damaged or diseased tissues during a point of care procedure. This surgical method of performing "personal cell therapy" (PCT) provides the physician with an advanced alternative method to care for patients with arthritic conditions. Many current therapeutic endeavors are aimed at mitigating symptomatic pain or reducing the secondary inflammatory response (i.e. prostaglandin formation) to limit pain in hopes of giving the body time to "heal its injury." By initially providing SVF, rich in stem cells, it is likely that the healing cells will respond to the cytokine response from the injury in order to effect primary healing. With new technology, there typically comes skepticism from both patients and referring physicians. As such, it's understandable that the majority of patients currently seeking investigative personal cell therapy will exhibit later stage chronic conditions that are more difficult to repair than those treated closer to the acute phase. Ultimately, as cell therapy gains widespread acceptance, it is likely that there will be a greater tendency toward cell deployment closer to the acute phase of injury in order to achieve more optimal results.

\section{Author Contributions}

All authors provided substantial contributions to the conception and design, acquisition of data, or analysis and interpretation of data; drafted the article or revised it critically for important intellectual content; have final approval of the version of the article to be published; and agree to be accountable for all aspects of the work in ensuring that questions related to accuracy or integrity of any part of the work are appropriately investigated and resolved.

\section{Acknowledgements}

Shawntae Dowell spent many hours compiling the data and providing statistical analysis. Sean Berman contributed considerable time adding graph material, helping review and revise the manuscript.

Below is a list of members of the Cell Surgical Network that have contributed to this study. All members have contractually agreed to participate in the registration of patients through the CSN database and agreed to publication.

Contributions made by members of the Cell Surgical Network.

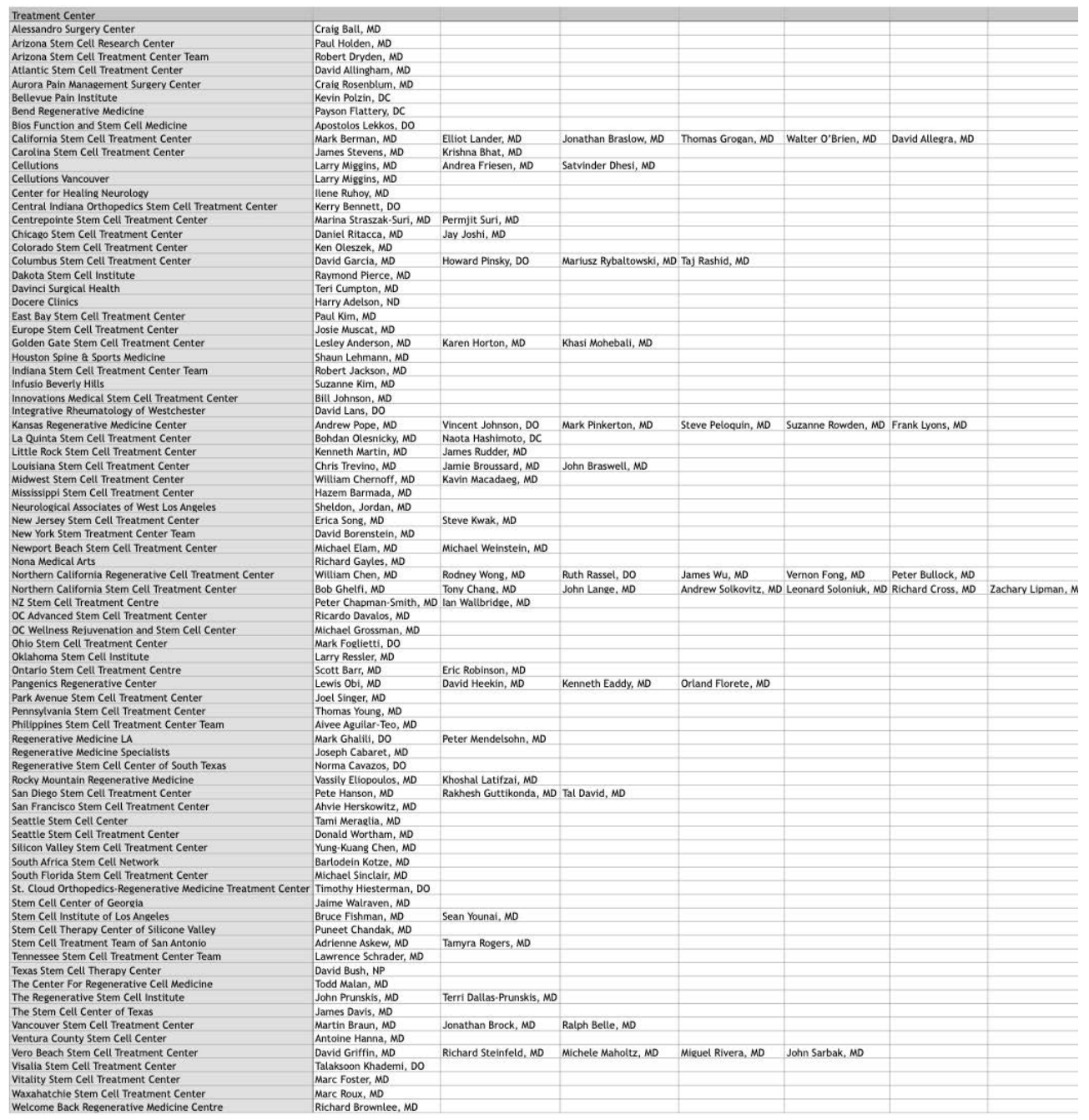




\section{References}

1. Michalek J, Moster R, Lukacl L, Kenneth Proefrock, Miron Petrasovic, et al. (2017) Stromal vascular fraction cells of adipose and connective tissue in people with osteoarthritis: A case control prospective multi-centric non-randomized study. Global Surgery 3: 1-9.

2. Centeno CJ, Al-Sayegh H, Freeman MD, Smith J, Murrell WD (2016) A multi-center analysis of adverse events among two thousand, three hundred and seventy two adult patients undergoing adult autologous stem cell therapy for orthopaedic conditions. Int Orthop 40: 1755-1765.

3. Pak J (2011) Regeneration of human bones in hip osteonecrosis and human cartilage in knee osteoarthritis with autologous adipose-tissue-derived stem cells: A case series. J Med Case Rep 5: 296.

4. Fodor P, Paulseth SPT (2016) Adipose Derived Stromal Cell (ADSC) injections for pain management of osteoarthritis in the human knee joint. Aesthetic Surgery Journal 36: 229-236.

5. Koga H, Engebretsen L, Brinchmann JE, Muneta T, Sekiya I (2009) Mesenchymal stem cell-based therapy for cartilage repair: A review. Knee Surg Sports Traumatol Arthrosc 17: 1289-1297.

6. Harman R, Carlson K, Gaynor J, Gustafson A, Dhupa S, et al. (2016) A prospective, randomized, masked and placebo-controlled efficacy study of intra-articular of allogeneic adipose stem cells for treatment of osteoarthritis in dogs. Front Vet Sci 3: 81.

7. Augello A, Tasso R, Negrini SM, Cancedda R, Pennesi G (2007) Cell therapy using allogeneic bone marrow mesenchymal stem cells prevents tissue damage in collagen-induce arthritis. Arthritis Rheum 56: 1175-1186.

8. Gonzalez-Rey E, Gonzalez MA, Varela N, O’Valle F, Hernandez-Cortes P, et al. (2010) Human adipose-derived mesenchymal stem cells reduce inflammatory and $T$ cell responses and induce regulatory $T$ cells in vitro in rheumatoid arthritis. Ann Rheum Dis 69: 241-248.

9. Chen FH, Tuan RS (2008) Mesenchymal stem cells in arthritic diseases. Arthritis Res Ther 10: 223.

10. Zheng ZH, Li XY, Ding J, Jia JF, Zhu P (2008) Allogeneic mesenchymal stem cell and mesenchymal stem cell-differentiated chondrocyte suppress the responses of type II collagen-reactive $\mathrm{T}$ cells in rheumatoid arthritis. Rheumatology 47: 22-30.

11. Ryan JM, Barry FP, Murphy JM, Mahon BP (2005) Mesenchymal stem cells avoid allogeneic rejection. J Inflamm
(Lond) 2: 8.

12. Ryan AE, Lohan P, O'Flynn L, Treacy O, Chen X, et al. (2014) Chondrogenic differentiation increase anti-donor immune response to allogeneic mesenchymal stem cell transplantation. Mol Ther 22: 655-667.

13. Holmqvist AS, Chen $Y$, Wu J, Battles K, Bhatia R, et al. (2018) Assessment of late mortality risk after allogeneic blood or marrow transplantation performed in childhood. JAMA Oncology 4: e182453.

14. Sanz J, Cano I, González-Barberá EM, Arango M, Reyes J, et al. (2015) Bloodstream infections in adult patients undergoing cord blood transplantation from unrelated donors after myeloablative conditioning regimen. Biol Blood Marrow Transplant 21: 755-760.

15. Parody R, Martino R, Rovira M, Vazquez L, Vázquez MJ, et al. (2006) Severe infections after unrelated donor allogeneic hematopoietic stem cell transplantation in adults: Comparison of cord blood transplantation with peripheral blood and bone marrow transplantation. Biol Blood Marrow Transplant 12: 734-748.

16. Yazaki M, Atsuta $\mathrm{Y}$, Kato $\mathrm{K}$, Kato $\mathrm{S}$, Taniguchi $\mathrm{S}$, et al. (2009) Incidence and risk factors of early bacterial infections after unrelated cord blood transplantation. Biol Blood Marrow Transplant. 15: 439-446.

17. Narimatsu $H$, Matsumura $T$, Kami M, Miyakoshi $S$, Kusumi $\mathrm{E}$, et al. (2005) Bloodstream infection after umbilical cord blood transplantation using reduced-intensity stem cell transplantation for adult patients. Biol Blood Marrow Transplant 11: 429-436.

18. Tomonari A, Takahashi S, Ooi J, Tsukada N, Konuma T, et al. (2007) Bacterial bloodstream infection in neutropenic adult patients after myeloablative cord blood transplantation: Experience of a single institution in Japan. Int J Hematol 85: 238-241.

19. Kilinc MO, Santidrian A, Minev I, Toth R, Draganov D, et al. (2018) The Ratio of ADSCs to HSC-progenitors in adipose tissue derived SVF may provide the key to predict the outcome of stem cell therapy. Clin Transl Med 7: 5.

20. Berman, Lander (2017) A prospective safety study of autologous adipose stromal vascular fraction using a specialized surgical processing system. American J Cosm Surg 2017, $1-14$.

21. Yoshimura K, Shigeura T, Matsumoto D, Sato T, Takaki Y, et al. (2006) Characterization of freshly isolated and cultured cells erived from the fatty and fluid portions of liposuction aspirates. J Cell Physiol 208: 64-76. 